\title{
Tackling the infrared problem of thermal QCD
}

MIT-CTP-3421

\author{
Y. Schröder ${ }^{\text {a* }}$ \\ ${ }^{a}$ Center for Theoretical Physics, MIT, Cambridge, MA 02139, USA
}

Perturbative calculations of corrections to the behavior of an ideal gas of quarks and gluons, the limit that is formally realized at infinite temperature, are obstructed by severe infrared divergences. The limits to computability that the infrared problem poses can be overcome in the framework of dimensionally reduced effective theories. Here, we give details on the evaluation of the highest perturbative coefficient needed for this setup, in the continuum.

\section{INTRODUCTION}

The theory of strong interactions, Quantum Chromodynamics (QCD), is guaranteed to be accessible to perturbative methods once one of its parameters, the temperature $T$, is increased towards asymptotically high values. This general statement relies solely on the well-known property of asymptotic freedom.

In practice, however, calculations of corrections to the behavior of an ideal gas of quarks and gluons, the limit that is formally realized at infinite $T$, are obstructed by severe infrared (IR) divergences [1: for every observable, there exists an order of the perturbative expansion to which an infinite number of Feynman diagrams contribute. No method is known how to re-sum these infinite classes of diagrams, a fact that seriously obstructs progress in the field of thermal QCD.

It is known how to evade this obstruction using dimensionally reduced effective theories. The key idea is to map the infrared sector of thermal QCD onto a three-dimensional pure gauge theory [1234, whose contribution, being a pure number, could be extracted numerically by MonteCarlo simulations. While the expansion of the QCD pressure in the effective theory framework, up to the order where IR contributions are relevant, is now known analytically [4, realizing the numerical extraction of the yet-unknown number emerging from the IR sector is a challenging open problem, with the main complication that highorder matching between lattice and continuum regularization schemes is necessary [5].

*talk presented at Lattice 2003.

\section{SETUP}

Let us now switch gears and focus on one of the main building blocks of the procedure, while for a detailed description of the setup as well as notation and further references, we refer to [4. In particular, we want to compute the (negative) $3 \mathrm{~d}$ vacuum energy density of a pure $\mathrm{SU}(\mathrm{N})$ gauge theory,

$$
\lim _{V \rightarrow \infty} \frac{1}{V} \ln \int \mathcal{D} A_{i} \exp \left(-\int \mathrm{d}^{d} x \frac{1}{2} \operatorname{Tr} F_{i j}^{2}\right),
$$

which in a weak-coupling expansion can be written as the sum of all connected vacuum graphs containing gluons and ghosts. Since the theory is confining, the computation involves IR divergent integrals (starting at the 4-loop level here), forbidding a perturbative evaluation of the full vacuum energy. One can however obtain its logarithmic ultraviolet divergence.

Note that in $3 \mathrm{~d}$ the coupling constant $g$ is dimensionful, hence the full answer must be of the form

$d_{A} C_{A}^{3} \frac{g^{6}}{(4 \pi)^{4}}\left[\alpha_{\mathrm{G}}\left(\frac{1}{\epsilon}+8 \ln \frac{\bar{\mu}}{2 m_{\mathrm{M}}}\right)+\beta_{\mathrm{G}}+\mathcal{O}(\epsilon)\right]$,

where $m_{\mathrm{M}} \equiv C_{A} g^{2}$ is a dynamically generated infrared scale in the confining theory, and $C_{A}=N$ and $d_{A}=N^{2}-1$ are the Casimir and the dimension of the adjoint representation, respectively. Because of super-renormalizability, the coefficient $\alpha_{\mathrm{G}}$ can then be computed in 4-loop perturbation theory, even if the constant part $\beta_{\mathrm{G}}$ cannot.

If we just carry out the 4-loop computation in strict dimensional regularisation, the result vanishes, because there are no perturbative mass 
scales in the problem. This means that UV and IR divergences (erroneously) cancel against each other. Therefore, we have to be more careful in order to determine $\alpha_{\mathrm{G}}$. To regulate the IR divergences, we introduce by hand a mass scale, $\mathrm{m}^{2}$, into the gauge field (and ghost) propagators. One has to keep in mind, however, that now only the coefficient $\alpha_{\mathrm{G}}$ multiplying $1 / \epsilon$ is physically meaningful, as it contains the desired gauge independent ultraviolet divergence. On the contrary, the constant part depends on the gauge parameter $\xi$, because the introduction of $m^{2}$ breaks gauge invariance, and has nothing to do with $\beta_{\mathrm{G}}$.

Note that e.g. diagrams with self-energy insertions can have IR sub-divergences, since IR divergences are known to be present in the $3 \mathrm{~d}$ 2-loop gluon propagator. To avoid the problem of overlapping IR divergences from the outset, we have hence chosen to employ the mass parameter rigorously, i.e. by rewriting every $1 / p^{2}$ as $1 /\left(p^{2}+m^{2}\right)$.

This leaves us within the class of fully massive integrals. The computation can be divided in three parts. Roughly, those are (1) diagram generation [6], specification of Feynman rules and color algebra, (2) reduction to master integrals 78, (3) expansion in $d=3-2 \epsilon$ dimensions.

We will refrain from commenting on the first two parts of the computation here, since they are well documented in the references given above. Due to the complexity of the computation, both steps are automatized, allowing for the handling of a large set of diagrams.

\section{MASTER INTEGRAL REPRESEN- TATION}

Let us now give a little more detail on part (3) of the computation. At this point, all diagrams are expressed in terms of 19 scalar master integrals, which are enumerated in [8]. The general structure is

$d_{A} C_{A}^{3} \frac{g^{6}}{(4 \pi)^{4}} \sum_{i=1}^{19} \frac{\operatorname{poly}_{i}(d, \xi)}{\operatorname{poly}_{i}(d)} \operatorname{Master}_{i}(d)$,

where $d$ is still an arbitrary (space-time) dimension. Only now do we need to specify $d=3-2 \epsilon$.

While it is trivial to expand the polynomial prefactor in $\epsilon$, considerable effort has to be put into obtaining the expansion for the master integrals to the depth required. Since we need the $\epsilon$-poles only, it would seem sufficient to compute the divergent parts of all master integrals. It turns out, however, that the prefactor develops poles as well around 3 dimensions, having terms proportional to $1 /(d-3)$ multiplying 10 of the master integrals, and even double poles in 4 of those cases.

A crucial simplification can be made by exploiting the freedom of choosing the basis of master integrals to represent the sum of diagrams Eq. (2). Going back to the tabulated relations between integrals that were derived by partial integration and used in part (2), we found two most useful relations:

$$
\begin{aligned}
& \text { (b) }=-\frac{8(d-3)}{5} \text {. } \\
& -\frac{(d-3)(3 d-8)}{5} \bigcirc \\
& +\frac{(2 d-7)(2 d-5)}{25} \bigcirc \\
& -\frac{(d-2)^{2}}{10}(\bigcirc)^{2} \bigcirc \text {, } \\
& \theta=-\frac{2}{3} \theta-\frac{3 d-10}{6} \otimes \\
& +\frac{1}{3} \bigcirc+\frac{d-3}{9} \circlearrowright \text {. }
\end{aligned}
$$

Notation: each line represents a massive scalar propagator, a dot on a line means an extra power, vertices have no structure. Trading the two master integrals on the lhs of the above equations for the first ones on the rhs respectively (all others are already included in the basis), the $d$ dimensional representation Eq. (2) of course still holds, albeit with a 'primed' version of the basis,

$d_{A} C_{A}^{3} \frac{g^{6}}{(4 \pi)^{4}} \sum_{i=1}^{19} \frac{\operatorname{poly}_{i}^{\prime}(d, \xi)}{\operatorname{poly}_{i}^{\prime}(d)} \operatorname{Master}_{i}^{\prime}(d)$.

In this new basis, none of the prefactors has a double pole in $3 \mathrm{~d}$, while only 7 members of the new 'primed' basis are multiplied by a single pole. It is not excluded that there exists a choice of basis for which the prefactors never get singular, but this choice is currently not known to us. 


\section{EXPANSION}

It turns out that (almost) all integrals are known analytically to the order needed for obtaining the poles in the sum of all diagrams. Lower loop cases have been treated in [10, while analytic results for the divergences of all $3 \mathrm{~d}$ 4-loop master integrals as well as numerical and some analytic results for their constant parts as well as the $\mathcal{O}(\epsilon)$ term of the 2-loop sunset integral can be found in 11. By an amusing relation specific to $3 \mathrm{~d}$, namely the fact that the leading term of the 3d 1-loop scalar 2-point integral is an arctan, whose derivative with respect to a mass looks like a propagator with double mass, it is furthermore possible to relate the leading term of one of the 4-loop master integrals to a 3-loop case [12:

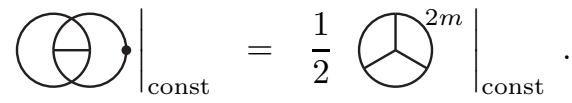

There are however 2 master integrals (out of the 7 which get multiplied by a $1 / \epsilon$ from the prefactor) whose constant term we do not yet know analytically. Let us denote their leading parts by $x_{2}$ and $x_{3}$ (by naive power-counting, it is easy to see that both are UV finite),

$\Theta=x_{2}+\mathcal{O}(\epsilon) \quad,=x_{3}+\mathcal{O}(\epsilon)$

Filling in the known expansions for the master integrals as well as expanding the prefactors, higher poles cancel in the sum of diagrams, and we are left with a single pole only:

$d_{A} C_{A}^{3} \frac{g^{6}}{(4 \pi)^{4}}\left(\frac{\bar{\mu}}{2 m}\right)^{8 \epsilon}\left(\frac{p(\xi)}{\epsilon}+\mathcal{O}\left(\epsilon^{0}\right)\right)$.

The polynomial $p$ is of order 6 in the gauge parameter $\xi$ and contains, besides a collection of numbers like $\pi^{2}, \ln 2$ and dilogarithms, the two unknowns $x_{2}$ and $x_{3}$. Clearly, in order for the result to be gauge independent, all $\xi$-dependence has to vanish once $x_{2}$ and $x_{3}$ are known. We can now reverse the argument and try to fix these constants by requiring gauge independence. Inspecting the polynomial, it turns out to have a very simple structure:

$p(\xi)=\alpha_{G}+\left(x_{2}-6 x_{3}-b\right) \sum_{i=0}^{6} c_{i} \xi^{i}$
$\alpha_{G}=\frac{43}{96}-\frac{157}{6144} \pi^{2} \approx 0.195715 \ldots$,

where the $c_{i}$ are pure numbers and $b=\operatorname{Li}_{2} \frac{1}{4}+$ $\operatorname{Li}_{2} \frac{1}{5}-3 \operatorname{Li}_{2} \frac{2}{5}+2(\ln 2)^{2}-\frac{3}{2}(\ln 3)^{2}-(\ln 5)^{2}-$ $2 \ln 2 \ln 5+3 \ln 3 \ln 5+\frac{\pi^{2}}{8}$. We have checked by numerical integration that

$x_{2}-6 x_{3}=b \approx-0.00200966335 \ldots$

to nine significant digits, hence establishing Eq. (10) as our main result for the logarithmic divergence of $3 \mathrm{~d}$ pure gauge theory.

Acknowledgments I would like to thank K. Kajantie, M. Laine and A. Vuorinen for numerous valuable discussions on the matter presented here, and KK and ML for an independent check of Eq. (11). This work was supported in parts by the DOE, under Cooperative Agreement no. DF-FC02-94ER40818.

\section{REFERENCES}

1. A.D. Linde, Phys. Lett. B 96 (1980) 289; D.J. Gross, R.D. Pisarski and L.G. Yaffe, Rev. Mod. Phys. 53 (1981) 43.

2. P. Ginsparg, Nucl. Phys. B 170 (1980) 388; T. Appelquist and R.D. Pisarski, Phys. Rev. D 23 (1981) 2305.

3. E. Braaten and A. Nieto, Phys. Rev. Lett. 76 (1996) 1417.

4. K. Kajantie, M. Laine, K. Rummukainen and Y. Schröder, Phys. Rev. D 67 (2003) 105008.

5. F. DiRenzo et. al., these proceedings.

6. K. Kajantie, M. Laine and Y. Schröder, Phys. Rev. D 65 (2002) 045008.

7. S. Laporta, Int. J. Mod. Phys. A 15 (2000) 5087.

8. Y. Schröder, Nucl. Phys. Proc. Suppl. 116 (2003) 402.

9. J.A.M. Vermaseren, math-ph/0010025

10. A.K. Rajantie, Nucl. Phys. B 480 (1996) 729; ibid. B 513 (1996) 761 (E); D. J. Broadhurst, Eur. Phys. J. C 8 (1999) 363.

11. K. Kajantie, M. Laine, K. Rummukainen and Y. Schröder, JHEP 0304 (2003) 036; A. Vuorinen, Master's Thesis, Helsinki University, 2001.

12. M. Laine, private notes. 